\title{
Obstetric outcome in pregnancies with borderline versus normal amniotic fluid index
}

\author{
Tony Augustine, Saphina Palakkan*, Umadevi N.
}

Department of Obstetrics and Gynecology, M. E. S. Medical College, Perinthalmanna, Kerala, India

Received: 02 June 2019

Accepted: 02 July 2019

\section{* Correspondence:}

Dr. Saphina Palakkan,

E-mail: drcamans@gmail.com

Copyright: $(\subset)$ the author(s), publisher and licensee Medip Academy. This is an open-access article distributed under the terms of the Creative Commons Attribution Non-Commercial License, which permits unrestricted non-commercial use, distribution, and reproduction in any medium, provided the original work is properly cited.

\section{ABSTRACT}

Background: Amniotic fluid is an indicator of placental function on the fetal development. It is regulated by several systems, including transmembraneous pathway, fetal production (fetal urine and lung fluid) and uptake (fetal swallowing), and the balance of fluid movement via osmotic gradients. The amniotic fluid index is the most commonly used method of measuring amniotic fluid. An AFI between 5 to $8 \mathrm{cms}$ is borderline oligohydraminos. To compare the fetal and maternal outcome in pregnancies with borderline amniotic fluid index ( 5 to $8 \mathrm{cms}$ ) and normal AFI.

Methods: This prospective cohort study was carried out on 94 pregnant patients attending the antenatal clinic of MES Medical College, Perinthalmanna between January $1^{\text {st }}$ and December $31^{\text {st }} 2016$. Women with a singleton pregnancy in the third trimester were enrolled into the study. Of these 47 cases were in the normal AFI group and 47 cases in the borderline group. Adequate information was obtained from the patient's medical record and the groups were compared on maternal and fetal complications.

Results: The study results showed that more cases with borderline AFI had their non stress test to be nonreactive (59.6\% versus $23.4 \%$ ) and was associated with more fetal heart rate abnormalities $(51 \%$ versus $17 \%)$. The number of meconium stained amniotic fluid (48.9\% versus $19.1 \%)$ and caesarean deliveries $(51.1 \%$ versus $21.2 \%)$ were also more among the borderline group.

Conclusions: Findings indicated that there are more adverse fetal and maternal outcome among the borderline AFI group.

Keywords: Borderline amniotic fluid index, Fetal outcome, Maternal outcome, Normal amniotic fluid index, Pregnancy outcome, Pregnancy complication

\section{INTRODUCTION}

Amniotic fluid is the fluid in the amniotic cavity which gives the protective environment for the fetus to nourish, breathe, utilize nutrients and grow. It has got dynamic physiology throughout pregnancy, enriching the fetal growth. It significantly affects the perinatal outcome, hence necessitates its measurement. Many methods are being practiced worldwide, with every method having both advantages and limitations.
Amniotic fluid regulation represents a complex interaction of many systems-fetal respiratory, cardiovascular and fluid balance, urinary tract, skin, gastrointestinal, neurology, placenta and maternal factors. Amniotic fluid functions can be categorized as physical, functional and homeostasis. Modern understandings of fetal urinary system, neurologic and placental impacts on amniotic fluid volume are supplemented by many researches. Proteomics reveals that there are multiple amniotic fluid compounds that can predict preterm 
labour, premature cervical effacement and fetal infection. $^{1}$ The inflammatory cascade leading to incompetence of cervix, preterm rupture of membranes and preterm delivery, all may begin in the first trimester, according to amniocentesis results. Sludge on ultrasound suggests inflammatory amniocytes accumulating as inappropriate cervical effacement begins. ${ }^{2}$ Several of the amniotic fluid defensive properties are significantly elevated in preterm labour. ${ }^{3}$

These qualitative changes are not detected just by measuring amniotic fluid volume on ultrasound-more research will determine the role of amniocentesis in managing preterm labour. Amniotic fluid assessment is an essential part of evaluation for antenatal fetal surveillance. ${ }^{4}$ The Amniotic Fluid Index (AFI) is the most common practical method for the estimation of amniotic fluid volume. ${ }^{5}$ The amniotic fluid index (AFI) has been one of the integral component of fetal assessment during antenatal ultrasound examination for over 20 years. Decreased amniotic fluid or oligohydraminos is defined as AFI less than $5 \mathrm{cms}$ which represents a value below the first percentile.The term borderline AFI or borderline oligohydraminos is controversial. Usually it is taken as AFI between 5 and $8 \mathrm{cms}$. Petrozella and colleagues 3 found that though pregnancies between 24 and 34 weeks were not more likely to be complicated by maternal hypertension, stillbirth or neonatal death compared to

patients with AFI more than $8 \mathrm{cms}$, they were reported to have higher rates of preterm delivery, caesarean delivery for a non reassuring fetal heart rate pattern and fetal growth restriction were found. In this study we assess the outcomes, both maternal and fetal in patients with borderline AFI at term.

\section{METHODS}

This was a Cohort study conducted in the Obstetrics and Gynecology department of a tertiary care hospital (M.E.S medical college, Perithalmanna) in south India after obtaining approval from the institutional Ethical Committee.

Cohort 1: Antenatal Women with Border line AFI who attended the antenatal clinic in the Department of Obstetrics and Gynecology at MES Medical College, Perinthalmanna between January $1^{\text {st }}$ and December $31^{\text {st }}$ in 2016.

Cohort 2: Antenatal Women with Normal AFI who attended the antenatal clinic in the Department of Obstetrics and Gynecology at MES Medical College Perinthalmanna between January $1^{\text {st }}$ and December $31^{\text {st }}$ in 2016.

\section{Inclusion criteria}

- Single live intrauterine gestation with vertex presentation.
- 37 completed weeks of gestation

- Intact membranes

- $\quad$ AFI between 5 and $18 \mathrm{cms}$.

\section{Exclusion criteria}

- AFI less than 5 and more than $18 \mathrm{cms}$

- Gestational age less than 37 completed weeks

- Ruptured membranes

- Malpresentations and multiple gestations

- High risk pregnancies.

The calculated sample size was 94 (normal AFI group 47; borderline AFI group - 47). All antenatal woman who attended the antenatal clinic in the Department of Obstetrics and Gynaecology, MES Medical College Perinthalmanna during the study period underwent a ultrasonography after 37 weeks of gestation. The AFI was calculated based on this scan. Depending on the AFI, patients were categorized into borderline AFI group (5 to $8 \mathrm{cms}$ ) and normal AFI group (9 to 18). Beginning enrollment from January 1st 2016, the first 47 antenatal women with borderline AFI and satisfying the inclusion exclusion criteria formed the Cohort 1 and the first 47 women with normal AFI formed the Cohort 2.

An informed written consent was obtained from those enrolled for the study. Patients selected for the study, when admitted in the labour room or in the ward underwent a detailed clinical examination and all the findings were documented. Ultrasonogram findings were recorded and if needed were repeated to know about the AFI and Biophysical Profile. On admission, Non Stress Test (NST) was done for all women in both groups. Patients in the ward with reactive NST were monitored with daily fetal movement counting and NST. They were induced based on the bishop score according to hospital protocol. Those patients who get into labour were monitored with continuous CTG and partogram. At 3 to 4 cms of cervical dilatation, ARM is done to know the colour of amniotic fluid. If clear, continuous CTG monitoring was done. If meconium stained amniotic fluid was noted, amnioinfusion was given with two pints of normal saline. If thick meconium persists or if there was an abnormal CTG tracing, the patients were taken up for caesarean section.

Labour was monitored with partogram. All newborns were attended by neonatologist and APGAR at birth and at 5 minutes were noted. Those babies requiring admission were admitted in the NICU. Following Outcome measures were studied

- Labour - induced/spontaneous

- Mode of delivery

- $\quad$ APGAR scores at $5 \mathrm{~min}$.

- Birth weight

- Admission to neonatal ICU. 


\section{Statistical analysis}

For the statistical analysis, the statistical software SPSS version 16.0 for windows (SPSS Inc., Chicago, IL, USA) was used. The difference in fetal outcome and maternal outcome was looked for using chi square test or FisherExact-Test. The difference in birth weight was looked for using t-test and APGAR score using Mann Whitney U test.

\section{RESULTS}

94 patients (normal AFI group - 47; borderline AFI group - 47) were studied. The age of the patients varied between 18 to 39 years .In the borderline AFI cohort 8 patients were in the 18-20 group, 21 patients in the $21-25$ age group, 10 in the 26-30 age group, 5 in the 31-35 age group,and 3 patients were above 35 years. There were 27 primigravida and 20 multigravida in the borderline group and in the normal AFI group the it was 19 and 28 respectively. Among the borderline group 25 patients completed 37 weeks, 12 patients completed 38 weeks and 6 patients completed 39 weeks and 4 patients crossed 40 weeks of gestation. In the control group, 8 patients completed 37 weeks, 7 patients completed 38 weeks and 18 patients completed 39 weeks and 14 patients crossed 40 weeks of gestation.

Table 1: Distribution of fetal heart pattern.

\begin{tabular}{|llll|}
\hline $\begin{array}{l}\text { Fetal heart } \\
\text { pattern }\end{array}$ & $\begin{array}{l}\text { Borderline } \\
\mathrm{N}=47\end{array}$ & $\begin{array}{l}\text { Normal } \\
\mathrm{N}=47\end{array}$ & $\begin{array}{l}\text { Total } \\
\mathrm{N}=94\end{array}$ \\
\hline Normal & $19(40.4 \%)$ & $38(80.6 \%)$ & 57 \\
\hline $\begin{array}{l}\text { Early } \\
\text { deceleration }\end{array}$ & $4(8.5 \%)$ & $1(2.1 \%)$ & 5 \\
\hline $\begin{array}{l}\text { Late } \\
\text { deceleration }\end{array}$ & $6(12.8 \%)$ & $5(10.6 \%)$ & 11 \\
\hline $\begin{array}{l}\text { Variable } \\
\text { deceleration }\end{array}$ & $18(38.2 \%)$ & $3(6.4 \%)$ & 21 \\
\hline
\end{tabular}

28 patients in the borderline group and 11 patients in the control group had non reactive NST. The test was reactive among 19 patients in the borderline group and 36 in the control group. Fetal distress was significantly more among the borderline group and signifies that with decreasing AFI, perinatal risks increases ( $p$ value $<0.001$ ).

There was significant difference in fetal heart pattern had significant difference between the two groups as variable deceleration and late deceleration were more in the borderline group as evident by $38.2 \%$ and $12.8 \%$ respectively (Table 1). The control group on the other hand had only $6.4 \%$ and $10.6 \%$ of variable and late deceleration respectively ( $\mathrm{p}$ value $<0.001$ ). This indicates that with amniotic fluid reduction fetal distress increases.

28 patients in borderline group were induced labour while 19 patients had spontaneous onset of labour. However in the control group, 24 patients were induced and 23 patients had spontaneous onset of labour. There was no significant relation between the AFI level and mode of labour (spontaneous/induced) ( $\mathrm{p}$ value $>0.05$ ).

Table 2: Distribution of patients based on birth weight.

\begin{tabular}{|llll|}
\hline $\begin{array}{l}\text { Birth weight } \\
(\mathrm{kg}) \text { group }\end{array}$ & $\begin{array}{l}\text { Borderline } \\
\mathbf{N}=47\end{array}$ & $\begin{array}{l}\text { Normal } \\
\mathbf{N}=47\end{array}$ & $\begin{array}{l}\text { Total } \\
\mathbf{N}=94\end{array}$ \\
\hline$<1.5$ & $1(2.1 \%)$ & 0 & 1 \\
\hline $1.6-2.0$ & $11(23.4 \%)$ & $1(2.1 \%)$ & 12 \\
\hline $2.1-2.5$ & $15(32.1 \%)$ & $3(6.3 \%)$ & 18 \\
\hline $2.6-3.0$ & $12(25.5 \%)$ & $26(48.9 \%)$ & 38 \\
\hline $3.1-3.5$ & $7(14.9 \%)$ & $12(25.5 \%)$ & 19 \\
\hline $3.6-4.0$ & $1(2.1 \%)$ & $3(6.3 \%)$ & 4 \\
\hline$>4.0$ & 0 & $2(4.2 \%)$ & 2 \\
\hline
\end{tabular}

$51.1 \%$ of patients in the borderline group underwent LSCS while $48.9 \%$ had vaginal delivery. But in the control group only $21.2 \%$ patients underwent LSCS and $78.7 \%$ patients had a vaginal delivery. This difference in propotion was found to be significant ( $p$ value $=0.003$ ) indicating that decrease in the amniotic fluid increases the chance of caesarean section because of having more fetal distress. The study showed there is no statistical difference in induction delivery interval among study and control groups as 33 patients delivered within 12 hours and 14 patients took more than 12 hours to deliver from the time of induction. In the control group 31 patients delivered within 12 hours and 16 patients delivered after 12 hours of induction. Chi square test gave a $\mathrm{p}$ value of $0.658(>0.05)$ which is insignificant.

The amniotic fluid was clear in $51.1 \%$ of the borderline group and $48.9 \%$ had meconium stained amniotic fluid. But only $19.1 \%$ of the control group had meconium stained amniotic fluid and $80.8 \%$ had clear amniotic fluid. There is significant association between colour of meconium and AFI (borderline/normal) $(p=0.002)$. This shows that there was increasing incidence of meconium with decreased liquor.

Birth weight data showed more number of patients in the borderline group with lower birth weight as compared to the control group. Among the borderline group there was only 1 newborn with birth weight less than $1.5 \mathrm{~kg}$ and 1 newborn in the $3.6-4 \mathrm{~kg}$ group. There were 11 newborns in the $1.6-2 \mathrm{~kg}$ category, 15 newborns in 2.1 to $2.5 \mathrm{~kg}$ group, 12 in the 2.6 to $3 \mathrm{~kg}$ group and 7 newborns in 3.1 to $3.5 \mathrm{~kg}$ group. In the control group there were $1,3,26$, 12,3 and 2 patients in the $1.6-2 \mathrm{~kg}, 2.1-2.5,2.6-3 \mathrm{~kg}, 3.1-$ $3.5,3.6-4 \mathrm{~kg}$ and more than $4 \mathrm{~kg}$ respectively (Table 2 ). The $\mathrm{p}$ value was $<0.001 \quad(<0.05)$ which is significant showing decreased liquor has an association with lower birth weight of the infants.

The study showed no complications in the immediate postpartum period and postnatal/post operative period amon 87 patients. In the borderline 2 patients had fever, 2 
patients had wound gaping while in the control group 1 patient each had fever, paralytic ileus and wound gaping. On the whole there was no statistical difference as the $p$ value was $0.056(>0.05)$ which signifies AFI solely does not affect the maternal morbidity.

In the borderline group, 32 newborns had APGAR less than 7 and 15 had an APGAR score more than 7, while the control group had 37 newborns with APGAR less than 7 and 10 had APGAR more than 7. Calculating the chi square test, the $\mathrm{p}$ value was more than 0.05 , which means it is not significant and brings to the conclusion that no difference in the fetal outcome between borderline and control group. Newborn admissions were significantly higher among the study group as out of the total 17 NICU admissions in the study $27.6 \%$ were from the borderline group and only 4 infants from the control group got admitted in the NICU. The statistical test showed a $\mathrm{p}$ value of $0.037(<0.05)$ which is significant. This implies that with decreasing AFI, perinatal risk increases evident from the fact that more infants among the borderline group needed NICU care.

\section{DISCUSSION}

Amniotic fluid is the protective milieu which nourishes the fetus and helps in the process of labour. It helps the fetus to maintain the acid base balance so that fetus does not develop distress. With decreasing amniotic fluid the fetus is more likely to have increased perinatal risk.

In this study, perinatal risk is assessed with respect to decreasing amniotic fluid level, measured as amniotic fluid index (AFI) and borderline AFI is taken as AFI between 5 and $8 \mathrm{cms}$ and its impact on perinatal outcome and indirectly maternal outcome are studied. Phelan et al studied about the amniotic fluid index measurements during pregnancy and reported on its usefulness in assessing the fetal surveillance throughout pregnancy. ${ }^{6}$ Amniotic fluid index is a useful screening test for detecting intrapartum fetal distress and helps in taking early intervention needed to deliver an infant with good APGAR score. Collen and Mark et al studied the impact of amniotic fluid volume, assessed intrapartum, on the perinatal outcome and concluded that it is good in predicting the risk. ${ }^{7}$

In our study, both the groups when compared to age distribution, mean age, and parity no significant difference was seen. A similar study conducted by Kumar A et al, which also had similar results. ${ }^{8}$ Our study revealed that non reactive NST was more in the borderline group (59.6\%) against the normal group $(23.4 \%)$. Erika et al, also concluded in their study that borderline AFI had 2 fold increase in adverse perinatal outcome with regard to non reactive NST. ${ }^{9}$

In the present study the fetal heart pattern showed non reassuring pattern (late and variable deceleration) in almost $51 \%$ of the borderline group compared to the normal group. Jeng et al observed non reassuring fetal heart tracings for which caesarean deliveries were carried out and David et al in their study also observed decelerations in borderline AFI group and 2 fold increase in perinatal risk. ${ }^{10,11}$ Our study also compared the outcome based on the onset of labour, whether it was induced or spontaneous, and there was no significant difference between the AFI levels and mode of onset of labour. A study conducted by Mahapatro AK et al that compared the outcome following vaginal misoprostol induction in oligohydraminos and borderline oligohydraminos patients was not associated with an increased risk of cesarean section or instrumental vaginal delivery and intrapartum fetal distress. ${ }^{8}$ The mode of delivery had significant difference among the borderline and control group as indicated by $51.1 \%$ patients in the borderline group undergoing LSCS, compared to $21.2 \%$ of the normal group. Chauhan et al demonstrated an association between reduced AFI and increased risk of caesarean delivery12. Robson et al observed caesarean section incidence for fetal distress in eight of 14 patients (57\%) with AFI $<6.2$ cms13. Kwon and colleagues studied 217 pregnancies and found to have 3 times more caesarean section among the borderline AFI group14. The colour of amniotic fluid when compared showed that there is more incidence of meconium stained amniotic fluid among the borderline group $(48.9 \%)$ than the normal group (19.1\%). The study conducted by Rutherford et al also came to the same results that low levels of AFI are associated with more meconium stained amniotic fluid15.

Comparing the APGAR scores of the borderline group with the normal AFI group, no significant difference was noted and the $\mathrm{p}$ value was more than 0.05.A similar study conducted by Chauhan et al came to the conclusion that there was no increased incidence of low APGAR scores between borderline group and normal AFI group12. NICU admission was also studied which showed $27.1 \%$ of the borderline group requiring NICU admissions compared to the $4 \%$ from the normal group. Maryam Asgharnia et al 16 an Iranian study observed that incidence of low apgar $(<7$ at $5 \mathrm{~min})$ and NICU admission were more in the low AFI group. Kreiser et al 17 also studied the effect of borderline AFI and observed that more NICU admissions had come from the borderline group.

Another observation that was seen in the present study was the low birth weight which was more in the borderline group compared to the normal group. Gummus et al 18 studied 90 pregnancies with AFI between 5 and $10 \mathrm{cms}$ and found an approximately 2 fold increased risk for preterm birth, intrapartum fetal distress and birth weight below 10th percentile.

\section{CONCLUSION}

Reduced liquor detected during the antenatal period is associated with increased perinatal complications. 
Patients with borderline AFI should be under close surveillance during the antepartum and intrapartum period due to the increased chance of adverse outcome seen in them.

\section{Funding: No funding sources}

Conflict of interest: None declared

Ethical approval: The study was approved by the Institutional Ethics Committee

\section{REFERENCES}

1. Buhimschi CS, Weiner CP, Buhimschi IA: Proteomics part II: the emerging role of proteomics over genomics in spontaneous preterm labor/birth. Obstet Gynecol Surg. 2006;61(8):543-53.

2. Bujold E, Pasquier JC, Simoneau J. Intra-amniotic sludge, short cervix and risk of preterm delivery. J Obstet Gynecol Can. 2006;28(3):198-202.

3. Vogel I, Thorsen P, Curry A. Biomarkers for the prediction of preterm delivery. Acta Obstet Gynecol Scand. 2005;84(6):516-25.

4. Casey BM, McIntire DD, Bloom SL, Lucas MJ, Santos R, Twickler DM, et al. Pregnancy outcomes after antepartum diagnosis of oligohydramnios at or beyond 34 weeks' gestation. Am J Obstet Gynecol. 2000;182:909-12.

5. Phelan JP, Smith CV, Broussard P, Small M. Amniotic fluid volume assessment with the fourquadrant technique at 36-42 weeks' gestation. J Reprod Med. 1987;32:540-2.

6. Asgharnia M, Faraji R, Salamat F, Ashrafkhani B, Heirati SFD, Naimia S. Perinatal outcomes of pregnancies with borderline versus normal amniotic fluid index. Iran J Reproded. 2013;11(9):705-10.

7. Collen B, Morgan mark A, Garite TJ. The impact of amniotic fluid volume assessed intrapartum on perinatal outcome. Am J Obstet Gynecol. 1995;173: 167-74.

8. Mahapatro AK, Jena SK, Ghose S. Amniotic fluid index; Borderline liquor; Induction of labor; pregnancy outcome following induction of labour in oligohydramnios and borderline liquor at term: a comparative study. Fetal distress Oligohydramnios at Term. 2013;27:2826.

9. Chauhan SP, Sanderson M, Hendrix NW, Magann EF, Devoe LD. Perinatal outcome and amniotic fluid index in the antepartum and intrapartum periods: a meta-analysis. Am J Obstet Gynecol. 1999;181:1473-8.

10. Robson SC, Crawford RA, Spencer JA, Lee A. Intrapartum amniotic fluid index and its relationship to fetal distress. Am J Obstet Gynecol. 1992;166(1 Pt1):78-82.

11. Lagrew DC, Pircon RA, Nageotte M, Freeman RK, Dorchester W. How frequently should the amniotic fluid index be repeated?. Am J Obstet Gynecol. 1992;167(4):1129-33.

12. Chauhan SP, Taylor M, Shields D, Parker D, Scardo JA, Magann EF. Intrauterine growth restriction and oligohydramnios among high-risk patients. Am J Perinatol. 2007;24(04):215-21.

13. Robson SC, Crawford RA, Spencer JA, Lee A. Intrapartum amniotic fluid index and its relationship fetal distress. Am J Obstet Gynecol. 1992;166(1):7882.

14. Kwon JY, Kwon HS, Kim YH, Park YW. Abnormal Doppler velocimetry is related to adverse perinatal outcome for borderline amniotic fluid index during third trimester. J Obstet Gynaecol Res. 2006;32(6):545-9.

15. Moore TR. Clinical assessment of amniotic fluid in clinical obstetrics and gynecology.Pitkin Roy M, Scot James R. Philadelphia: Lippincot - Raven Publication. 1997;40(2):303-13.

16. Asgharnia M, Faraji R, Salamat F, Ashrafkhani B, Heirati SF, Naimian S. Perinatal outcomes of pregnancies with borderline versus normal amniotic fluid index. Iranian J Repro Med. 2013;11(9):705.

17. Kreiser D, El-Sayed YY, Sorem KA, Chitkara U, Holbrook RH, Druzin ML. Decreased amniotic fluid index in low-risk pregnancy. $\mathbf{J}$ Reprod Med. 2001;46:743-6.

18. Gumus II, Koktener A, Turhan NO. Perinatal outcomes of pregnancies with borderline amniotic fluid index. Arch Gynecol Obstet. 2007;276(1):17-9.

Cite this article as: Augustine T, Palakkan S,

Umadevi N. Obstetric outcome in pregnancies with borderline versus normal amniotic fluid index. Int $\mathrm{J}$ Reprod Contracept Obstet Gynecol 2019;8:3240-4. 